\title{
Molecular differences between stromal cell populations from deciduous and permanent human teeth
}

\author{
Nina Kaukua ${ }^{1,2}$, Mo Chen ${ }^{1}$, Paolo Guarnieri ${ }^{3}$, Markus Dahl' ${ }^{4}$, Mei Ling Lim ${ }^{5}$, Tülay Yucel-Lindberg ${ }^{4}$, Erik Sundström ${ }^{6}$, \\ Igor Adameyko ${ }^{7}$, Jeremy J Mao ${ }^{1}$ and Kaj Fried ${ }^{2 *}$
}

\begin{abstract}
Introduction: Deciduous and permanent human teeth represent an excellent model system to study aging of stromal populations. Aging is tightly connected to self-renewal and proliferation and thus, mapping potential molecular differences in these characteristics between populations constitutes an important task.

Methods: Using specifically designed microarray panels, Real-Time Quantitative Polymerase Chain Reaction (RT q-PCR), Western blot, immunohistochemistry and siRNA-mediated knock down experiments, we have detected a number of molecules that were differentially expressed in dental pulp from deciduous and permanent teeth extracted from young children and adults, respectively.

Results: Among the differentially regulated genes, high-mobility group AT-hook 2 (HMGA2), a stem cell-associated marker, stood out as a remarkable example with a robust expression in deciduous pulp cells. siRNA-mediated knock down of HMGA2 expression in cultured deciduous pulp cells caused a down-regulated expression of the pluripotency marker NANOG. This finding indicates that HMGA2 is a pulpal stem cell regulatory factor. In addition to this, we discovered that several proliferation-related genes, including CDC2A and CDK4, were up-regulated in deciduous pulp cells, while matrix genes COLIA1, fibronectin and several signaling molecules, such as VEGF, FGFr-1 and IGFr-1 were up-regulated in the pulp cells from permanent teeth.
\end{abstract}

Conclusions: Taken together, our data suggest that deciduous pulp cells are more robust in self- renewal and proliferation, whereas adult dental pulp cells are more capable of signaling and matrix synthesis.

\section{Introduction}

Senescence and aging are associated with the loss of self-renewing capacity of stem cells. This principle is valid for multiple locations in the body, including the nervous system, connective tissue and bone marrow, and plays a significant role in the regenerative potential of stem cells [1-3]. There are several extrinsic and intrinsic elements that contribute to the aging of stem cells. These include changes in the systemic environment through factors from the blood or modifications of the stem cell niche, and alterations of elements within the stem cells such as protein accumulation, damage to mitochondrial as well as nuclear DNA, telomere attrition

\footnotetext{
* Correspondence: kaj.fried@ki.se

${ }^{2}$ Department of Neuroscience, Karolinska Institutet, Stockholm SE-171 77, Sweden

Full list of author information is available at the end of the article
}

and cell cycle inhibition that eventually leads to failure of function [2,4]. Identification of potent tissue-specific stem cells and their banking is crucial for regenerative medicine. Teeth host pulpal stromal cells with subpopulations that have mesenchymal stem cell characteristics [5-7] that are easily extracted and amenable for manipulation. In animal models, these pulp cells seem to have a beneficial effect on spinal cord regeneration [8] and other types of trauma and disease, although their precise function is not clear and there is no evidence of any transdifferentiation into other cell types $[9,10]$. For reasons that remain elusive and contradictory to their apparent in vivo quiescence [11], dental pulp cells undergo rapid proliferation in ex vivo culture, apparently more rapid than bone marrow mesenchymal stem cells (BMMSCs) [5,12]. Previous work on genetic profiles of dental pulp cells has yielded several important clues 
[13-17]. Comparisons of gene expression between fast growing and slow growing pulp cell populations showed robust expression of transcription factors, E2F2, PTTG1, TWIST-1, and transcriptional cofactor, $L D B 2$, each with critical roles in cell growth and survival, in dental pulp cells, just as in the periodontal ligament and bone marrow [12].

Here, we address the issue of molecular differences between pulp cells from deciduous and permanent teeth to elucidate the mechanism that provides high stemness and self-renewal capacity. These two types of teeth offer an excellent model for studies of molecular differences between stromal cell populations, especially in relation to their age. We approached this task by comparing genetic profiles from cells isolated from different origins and generations of human teeth with subsequent functional studies. We found that the genetic profile of deciduous dental pulp cells differs from that of adult dental pulp cells, due to ontogenic discontinuity between deciduous and permanent teeth.

\section{Methods}

\section{Ethics statement}

Deciduous teeth and permanent teeth extracted for medical-dental reasons after thorough diagnoses by clinicians were collected from patients following approval by The Institutional Review Board at the Columbia University Medical Center and a Regional Ethical Committee in Stockholm. Patients provided informed consent to participate in this study. When deciduous teeth were obtained from children, informed consent was given from the next of kin, caretakers, or guardians on behalf of the children enrolled in our study. Informed consent was recorded, with age and gender of the donor and parent/patient approval but without any personal identifiers.

The Regional Ethical Committee in Stockholm approved the isolation and establishment of human embryonic stem cell lines. The HS181 cell line used in this study was derived at Karolinska Institutet, Karolinska University Hospital, Huddinge, Sweden.

The procedures for the use of human tissues, adult and embryonic gingiva, for experimental studies were approved by the Regional Ethical Committee, Stockholm.

\section{Cell isolation}

The deciduous teeth were collected from 3- to 12-year-old children $(n=8)$, and permanent teeth were collected from adults, 19 to 52 years of age $(n=8)$. Both deciduous and permanent teeth had full length roots with no visible root resorption and were extracted due to pericoronitis/periodontal issues, caries or orthodontic reasons. Cell isolation followed our previously described methods and approaches $[9,18]$. The tooth was mechanically separated into two halves to remove all soft tissue in the pulp chamber and the root canal. The pulp tissue was digested with $3 \mathrm{mg} / \mathrm{ml}$ collagenase I and $4 \mathrm{mg} / \mathrm{ml}$ dispase (Invitrogen,
Carlsbad, CA, USA) for one hour at $37^{\circ} \mathrm{C}$. The dissociated cells were passed through a $70-\mu \mathrm{m}$-cell strainer (BD Biosciences, San Jose, CA, USA) and plated in MEM-alpha medium (Invitrogen) containing 10\% fetal bovine serum (Gibco, Grand Island, NY, USA) and penicillin-streptomycin (Atlanta Biologicals, Lawrenceville, GA, USA). The cells from each pulp were cultured separately and then passaged at $90 \%$ confluency in different $10 \mathrm{~cm}$ dishes (Nunc, Rochester, NY, USA) or several wells of the six-well plate model (BD Biosciences) for expansion, and utilized for further analysis. The analyses were performed on pulp cells from different individual pulps without pooling.

\section{siRNA transfection}

Deciduous pulp cells were transfected with Dharmafect transfection reagent nr.4 (Thermo Scientific, Lafayette, CO, USA) according to the manufacturer's protocol, with a final concentration of $100 \mathrm{nM}$ siRNA ONTARGETplus Human HMGA2 and 100 nM siRNA ONTARGETplus Human Control Pool (Thermo Scientific), for 48 hours. The cells were transfected after one passage, at $40 \%$ confluency in $10 \%$ fetal bovine serum MEM-alpha medium containing no antibiotics.

\section{RNA and protein extraction}

RNA and protein were extracted from passage one cells with the Qiagen RNeasy Mini Kit (Valencia, CA, USA). At 90\% confluency, cells were washed with PBS (Lonza, Walkersville, MD, USA) and then placed in lysis buffer following the manufacture's protocols. For siRNA transfection experiments, total RNA was extracted according to the Qiagen AllPrep DNA/RNA/Protein Mini Kit protocol with a Qiagen AllPrep DNA/RNA/ Protein Mini Kit (Qiagen, Hilden, Germany).

\section{Microarray gene expression analysis}

Samples were analyzed using a PIQOR Stem Cell Microarray chip (Miltenyi Biotec, Auburn, CA, USA). This consisted of 942 relevant genes for stem/progenitor cells and key genes for cell differentiation to identify gene sets that are differentially expressed between dental pulp cells in the deciduous and adult teeth. All microarray data have been deposited in a public repository, Gene Expression Omnibus (GEO), with accession number GSE58668.

The isolated RNA was subjected to microarray analyses. A total of $1 \mu \mathrm{g}$ RNA for each sample was used for amplification and further analysis with the PIQOR stem cell microarray chip, followed by detection with a laser scanner (Agilent Technologies, Santa Clara, CA, USA). The dataset consisted of two microarrays, each containing 11 slides for a total of 16 samples. The Linear Model for Microarray Data package (Limma), within R/Bioconductor statistical framework, was used to pre-process the raw signal intensities, perform quality controls and estimate statistical significance 
[19]. Expression intensities were background-corrected using a convolution of normal and exponential distributions with an offset of 50, which were then normalized within every slide using LOESS normalization. We analyzed the red and green channels separately using the mixed model method and different groups were quantile-normalized separately. The correlation was computed between the two channels for the same spot. Differentially expressed genes for the eight different comparisons considered were identified by computing the contrasts, fitting to a linear model, performing the hypothesis tests and correcting for multiple testing using the Benjamin-Hochberg correction $(\mathrm{p} \leq 0.05)$. DAVID $[20,21]$ was used to test over-represented functional groups, and the output was further analyzed with Enrichment Map [22] a plugin for Cytoscape [23]. Additionally we used Ingenuity Pathway Analysis (IPA)(Ingenuity ${ }^{\circ}$ Systems) to generate the network of connections between modulated genes, using the knowledge-based topology. We then isolated the HMGA2 to carry out the causal network analysis with IPA.

\section{cDNA synthesis and real-time quantitative RT q-PCR} RNA was isolated with the Qiagen RNeasy Mini Kit (Qiagen). DNA contamination was eliminated with DNase treatment (Applied Biosystems, Foster City, CA, USA). cDNA was made according to, and with supplies from, Applied Biosystems. A real-time quantitative PCR procedure was performed with cDNA, Taqman Universal PCR mastermix and gene expression assays (Table 1) using the Applied Biosystems ViiA $7^{\mathrm{m}}$ Real-Time PCR System. Data were analyzed with the relative comparative method, where GAPDH was used to normalize the $\mathrm{C}_{\mathrm{t}}$ value. Deciduous dental pulp cells were set as control to calculate the differences from the permanent dental pulp cells.

For siRNA transfection experiments, cDNA synthesis was performed using the High Capacity cDNA Reverse Transcription Kit (Invitrogen, Foster City, USA) protocol. Further on, the RT q-PCR procedure was performed

Table 1 Primers for RT q-PCR

\begin{tabular}{llll}
\hline Gene symbol & Assay & Gene symbol & Assay \\
\hline GAPDH & Hs99999905_m1 & VEGF & Hs00900054_m1 \\
HMGA2 & Hs00171569_m1 & FGFR1 & Hs00241111_m1 \\
FABE & Hs02339439_g1 & PLXNA3 & Hs00250178_m1 \\
CDK1 & Hs00364293_m1 & TIMP1 & Hs99999139_m1 \\
MAD2L1 & Hs03063324_g1 & SERPINF1 & Hs01106937_m1 \\
MMP6 & Hs00757922_g1 & JMJ (Jarid2) & Hs01004457_m1 \\
CDK4 & Hs00364847_m1 & IGF1R & Hs00951562_m1 \\
MMP23A;B & Hs00270380_m1 & COL1A1 & Hs00164004_m1 \\
TK1 & Hs01062125_m1 & INHBA & Hs00170103_m1 \\
ITGB1 & Hs00559595_m1 & FN1 & Hs00277509_m1 \\
\hline
\end{tabular}

according to an Applied Biosystems protocol with gene expression assays GAPDH (FAM, Hs02758991_g1), NANOG (FAM, Hs02387400_g1), OCT4 (Hs03005111_g1) and HMGA2 (FAM, Hs00971725_m1) (Applied Biosystems, Foster City, CA, USA). PCR reactions were applied on ABI 7500 Fast- Real Time PCR System. As above, the relative comparative method was used to analyze data.

The cells from the HS181 cell line were maintained in knockout Dulbecco's modified Eagle's medium supplemented with $20 \%$ knockout serum replacement, $2 \mathrm{mM}$ glutamax, $0.5 \%$ penicillin-streptomycin, $1 \%$ non-essential amino acids, $0.5 \mathrm{mM}$ 2-mercaptoethanol and $8 \mathrm{ng} / \mathrm{ml}$ basic fibroblast growth factor (bFGF) (all from Life Technologies, Invitrogen, Stockholm, Sweden).

BMMSCs (Life Technologies, Invitrogen) at passage 3 were cultured in MesenPRO RS basal medium, supplemented with low serum of $2 \%$ MesenPRO RS growth supplement and $1 \%$ penicillin and streptomycin (Life Technologies, Invitrogen).

Total RNA was purified according to the manufacturer's instructions from deciduous pulp cells, HS181 and BMMSCs using a commercially available RNeasy Mini Kit (Qiagen, Germany). cDNA samples were prepared from $100 \mathrm{ng}$ of total RNA with superscript III (Invitrogen) using a Veriti thermal cycler (Applied Biosystems, Stockholm, Sweden). RT q-PCR was processed on a 7500 Fast RealTime PCR System (Applied Biosystems, USA) with Taqman Universal Master Mix and Taqman primer/probe for OCT4 (Hs03005111_g1), NANOG (Hs04260366_g1) and HMGA2 (Hs00971725_m1). The housekeeping gene, GAPDH (Hs02758991_g1) was used as an endogenous control. The expression level for each sample was normalized to $G A P D H$ and quantification of expression was estimated using the relative comparative method and was presented as relative fold change.

\section{SDS-PAGE and Western blot}

Proteins were isolated with the Qiagen RNeasy Mini Kit followed by acetone precipitation (deciduous pulps: $n=3$, permanent pulps: $n=3$ ). The protein extract was dissolved in $1 \%$ SDS. Protein concentration was measured with a Biorad RC DC Protein Assay Kit and SmartSpec Plus Spectrophotometer. A total of $20 \mu \mathrm{g}$ of protein was loaded into wells on NuPAGE ${ }^{\oplus}$ Novex $4 \%$ to $12 \%$ Bis-Tris Gels and then transferred onto polyvinylidene difluoride (PVDF) membranes (Biorad, Hercules, CA, USA) using electroblotting. The membranes were blocked with blocking buffer (Licor, Lincoln, NE, USA) and then probed with the antibodies for beta-actin (1:500, Santa Cruz Biotechnology, Santa Cruz, CA, USA), cdc2 (1:2000, Cell Signaling Technology, Danvers, MA, USA), Fatty Acid Binding Protein 5 (1 $\mu \mathrm{g} / \mathrm{ml}$, Abcam, Cambridge, MA, USA), HMGA2 (1:1000, Cell Signaling Technology) and PEDF (aka Serpinf1, $2 \mu \mathrm{g} / \mathrm{ml}$, Abcam) overnight in $4^{\circ} \mathrm{C}$ on a 
shaker. Thereafter, the corresponding secondary antibodies goat anti-mouse immunoglobulin G (IgG)-AP and goat anti-rabbit IgG-AP (Santa Cruz Biotechnology) were added for one hour at room temperature on a shaker. Proteins were detected using the 1-Step NBT/BC1P system (Thermo Scientific, Rockford, IL, USA). Quantification of the intensity levels of the Western blot bands was performed with ImageJ software.

\section{Immunohistochemistry}

Deciduous teeth were collected from 3- to 11-year-old patients $(\mathrm{n}=3)$ and permanent teeth were collected from
A

Deciduous vs Permanent pulp cells, experiment $\mathrm{nr}$ :

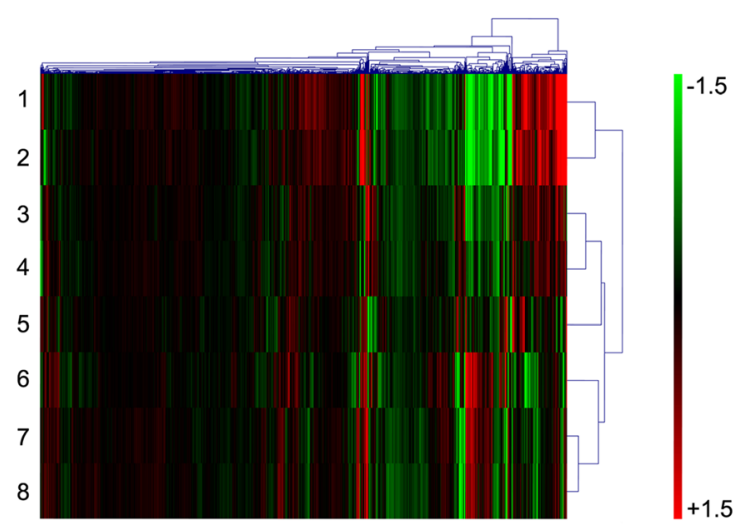

B

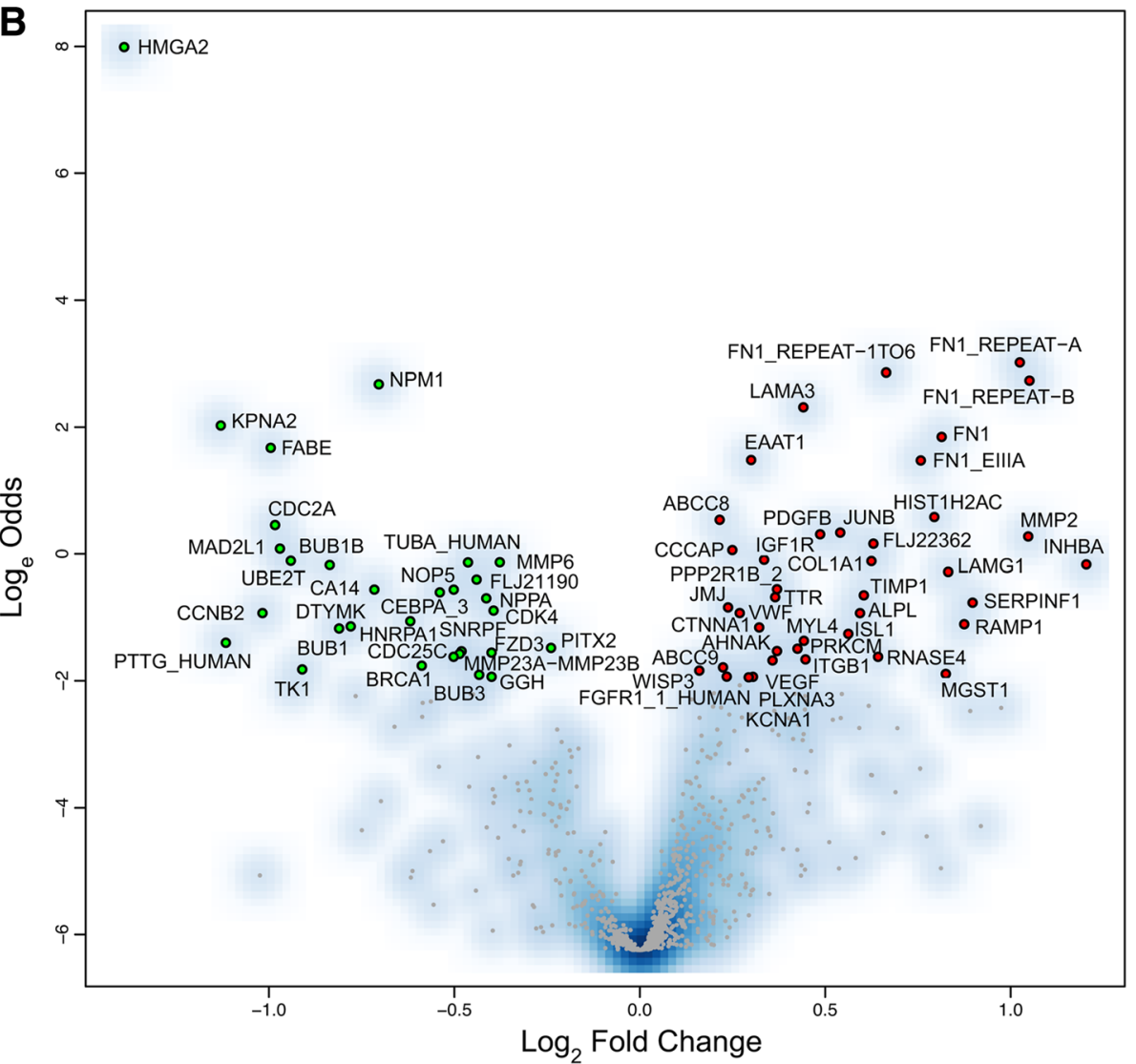

Figure 1 Heat map and volcano plot of genes in permanent versus deciduous pulp cells. A) Heat map of gene expression level in permanent pulp cells versus deciduous pulp cells. Each panel illustrates one comparison between permanent pulp cells and deciduous pulp cells from different subjects $(n=8)$. Red color indicates a high gene expression level, while green color means a low expression level in permanent compared to deciduous pulp cells. The data are z-score transformed. B) Volcano plot of up- and down-regulated genes in permanent versus deciduous pulp cells. Listed up- or down-regulated gene names have a significance of $P \leq .05$. 


\section{Table 2 Up-regulated genes in pulp cells from permanent teeth in microarray}

\begin{tabular}{|c|c|c|c|}
\hline Gene name & Reference sequence & Function & $\begin{array}{l}\log _{2} \text { fold } \\
\text { change }\end{array}$ \\
\hline INHBA & NM_002192 & Regulation of nerve cell survival, embryonic axial development and bone growth & 1.204 \\
\hline SERPINF1 & NM_002615 & Inhibits angiogenesis, is a neurotrophic factor involved in neuronal differentiation in retinoblastoma cells & 0.897 \\
\hline FN1 & $\begin{array}{l}\text { NM_002026,NM_212474, NM_212475, } \\
\text { NM_212476,NM_212478, NM_212482 }\end{array}$ & Cell adhesion, motility, opsonization, wound healing, and maintenance of cell shape & 0.814 \\
\hline COLIA1 & NM_000088 & Found in most connective tissues & 0.625 \\
\hline TIMP1 & NM_003254 & $\begin{array}{l}\text { Inhibitors of the matrix metalloproteinases, which are involved in degradation of the extracellular matrix. They } \\
\text { promote cell proliferation in a wide range of cell types. }\end{array}$ & 0.604 \\
\hline ITGB1 & $\begin{array}{l}\text { NM_002211,NM_033666, NM_033667,NM_033668, } \\
\text { NM_033669,NM_133376 }\end{array}$ & Receptors for collagen, fibronectin, fibrinogen and laminin & 0.447 \\
\hline VEGF & $\begin{array}{l}\text { NM_001025366,NM_001025367,NM_001025368, } \\
\text { NM_001025369 }\end{array}$ & Active in angiogenesis, vasculogenesis and endothelial cell growth & 0.358 \\
\hline IGFIR & NM_000875 & Binds insulin-like growth factor & 0.335 \\
\hline PLXNA3 & NM_017514 & Epithelial and neural tissue development & 0.293 \\
\hline FGFR1_1_HUMAN & $\begin{array}{l}\text { NM_015850,NM_023105, NM_023106,NM_023107 } \\
\text { NM_023108,NM_023110 }\end{array}$ & Interacts with fibroblast growth factors, influences mitogenesis and differentiation & 0.234 \\
\hline JMJ (Jarid2) & NM_004973 & Stem cell differentiation, negative regulation of cell proliferation & 0.238 \\
\hline
\end{tabular}


Table 3 Down-regulated genes in pulp cells from permanent teeth in microarray

\begin{tabular}{lll}
\hline Gene name & Reference sequence & Function \\
\hline HMGA2 & NM_003483 & Expressed during mammalian embryonic development. High expression in neural stem cells and decreases with age \\
FABE & NM_001444 & Found in epidermal cells, may be involved in keratinocyte differentiation \\
CDC2A & NM_001786, NM_033379 & Cell cycle key mediator of neuronal cell death in brain development and degeneration \\
MAD2L1 & NM_002358 & Cell division and mitosis \\
TK1 & NM_003258 & Cell division and mitosis \\
MMP23A-MMP23B & NM_006983, NR_002946 & Cytosolic enzyme, high in proliferating cells \\
FZD3 & NM_017412 & Involved in the breakdown of extracellular matrix \\
MMP6 & NM_005792 & Cell division \\
CDK4 & NM_000075 & Cell cycle \\
PTTG_human & NM_004219,NM_006607, NR_002734 & Cell division \\
\hline
\end{tabular}


adult patients ( 19 to 81 years of age, $n=3$ ). Teeth were cracked open and pulps were collected and snap frozen in optimal cutting temperature (OCT) cryomount (Histolab, Gothenburg, Sweden) for subsequent $5 \mu \mathrm{m} \mathrm{sec-}$ tioning. The sections were fixed for 20 minutes in $4 \%$ paraformaldehyde (Merck, Darmstadt, Germany), rinsed and then incubated overnight at room temperature with primary antibodies against HMGA2 (1:500, Cell Signaling Technology) and vimentin (1:200, Abcam). Additional staining was performed the following day with 4',6-diamidino-2-phenylindole (DAPI) (Invitrogen, Burlington, Canada) at $300 \mathrm{nM}$ applied for three minutes. Secondary antibodies used were Alexa Fluor 555 Donkey Anti-Rabbit $\operatorname{IgG}(\mathrm{H}+\mathrm{L})$ and Alexa Fluor ${ }^{\circ} 488$ Goat Anti-Chicken IgG $(\mathrm{H}+\mathrm{L})$ (Invitrogen, Eugene, OR, USA). Control sections were incubated where the primary antibody was omitted. This resulted in no detectable artifact. Additional negative control stainings were performed with isotype control for rabbit primary antibody (Invitrogen, Eugene, OR, USA), which resulted in no unspecific staining. Confocal microscopy was performed with Zeiss LSM700 CLSM and image processing was carried out with Imaris software.

\section{Hematoxylin \& eosin staining of deciduous and permanent tooth pulp}

Slides with pulp sections were washed in distilled water for one minute. They were then immersed in modified Mayers solution hematoxylin (Histolab, Gothenburg,
Sweden) for ten minutes, followed by rinsing in distilled water. Subsequently, slides were incubated in $0.2 \%$ eosin (Histolab) rinsed, dehydrated in graded steps of ethanol and xylene (VWR Chemicals, Fontenay-sous-Bois, France) and then mounted in Entellan (Merck). Light microscopy was performed with a Nikon Eclipse E600 microscope and images were processed in Adobe Photoshop CS5.

\section{Immunohistochemistry of embryonic and adult gingiva}

Human fetal lower jaw tissue (8.5 weeks after conception) was retrieved from elective routine abortions, with written consent from the pregnant women. The specimens $(n=2)$ were immersed in $4 \%$ paraformaldehyde (Merck) overnight and then rinsed in 30\% sucrose for 24 hours. They were then snap frozen in OCT cryomount (Histolab) for subsequent sectioning. Biopsies of human healthy gingiva from patients 54- to 67-years old $(n=3)$ were obtained with written consent from the donors, after approval by the Regional Ethical Committee, Stockholm. These samples were fixed in paraformaldehyde, rinsed, dehydrated and embedded in paraffin according to standard methods. After sectioning and deparaffination, they were processed for immunohistochemistry. Immunohistochemical staining was performed as described above with HMGA2 antibody (1:500, Cell Signaling Technology), CD31 antibody (1:300, Dako, Glostrup, Denmark) and Vectashield mounting medium with DAPI (Vector Laboratories, Burlingame, CA, USA). Imaging was performed using a

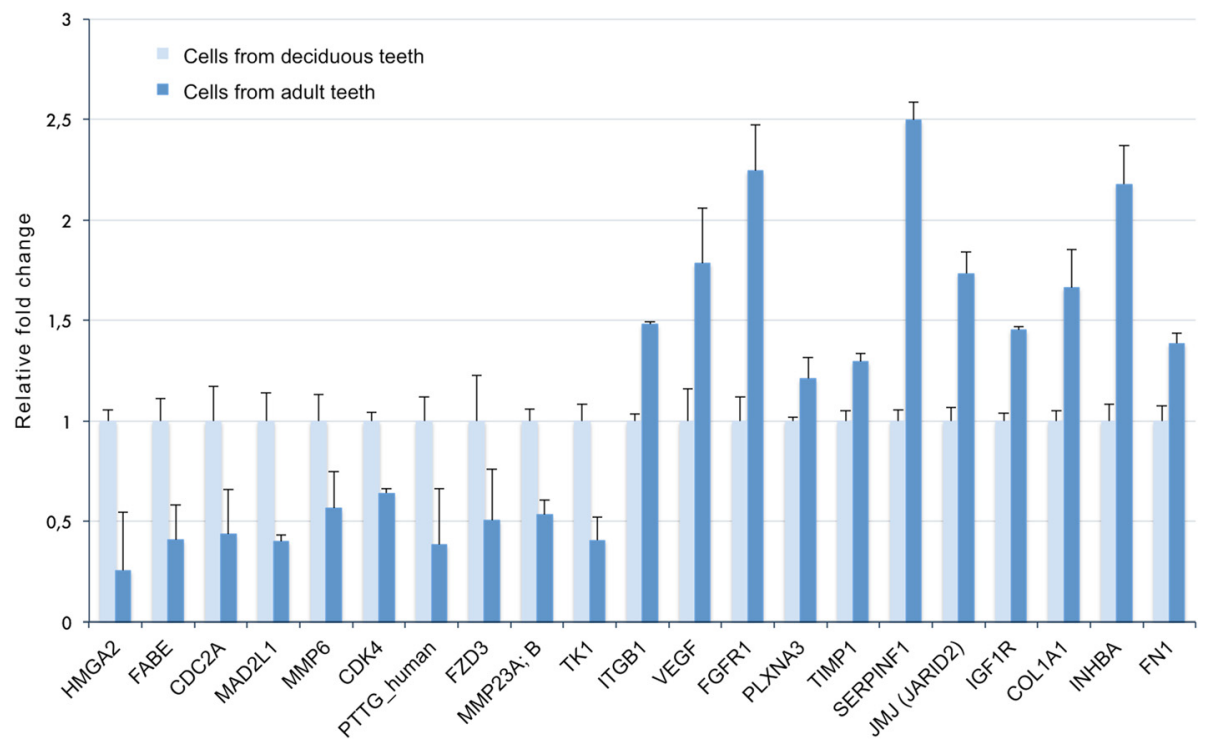

Figure 2 RT q-PCR of a subset of genes from the microarray. Results from RT q-PCR analysis of a subset of up- or down-regulated genes in permanent pulp cells versus deciduous pulp cells. The data agree with the results obtained in the microarray experiments, and were all significantly different, $P \leq 0.05$, Student's t-test. 
confocal microscope, Zeiss LSM710 CLSM, and image processing was carried out with Imaris software.

\section{Results}

Microarray gene expression analysis reveals differences between deciduous and permanent pulp cells

In order to tackle age differences in populations of connective tissue cells, we extracted cells from deciduous and permanent teeth obtained from young children and adults. Next we analyzed the transcriptomes from these cells using custom-generated microarray to discover differentially expressed transcripts related to stemness, proliferation and extracellular matrix production. We discovered genes that were differentially expressed by cells from deciduous versus adult teeth, as identified by cross-comparative analysis. Differences in hybridization intensities were observed for a proportion of the expressed genes as shown in the heat map (Figure 1A). The expression of a total set of 70 genes was significantly different $(P \leq 0.05$, Additional file 1$)$ and is shown in Figure 1B. Comparing deciduous to permanent teeth, 40 genes were up-regulated, and 30 genes were downregulated. Genes associated with cell division, extracellular matrix components, differentiation and aging differed remarkably between the deciduous and permanent pulp cells (Tables 2 and 3).

Genes encoding extracellular matrix proteins, including collagen type I (COL1A1) and fibronectin were differentially expressed in favor of pulp cells from permanent teeth. In addition, genes associated with cell differentiation were robustly expressed by pulp cells of the permanent teeth, including INHBA, SERPINF1, PLXNA3 and JMJ. In comparison, genes promoting cell proliferation, mitosis

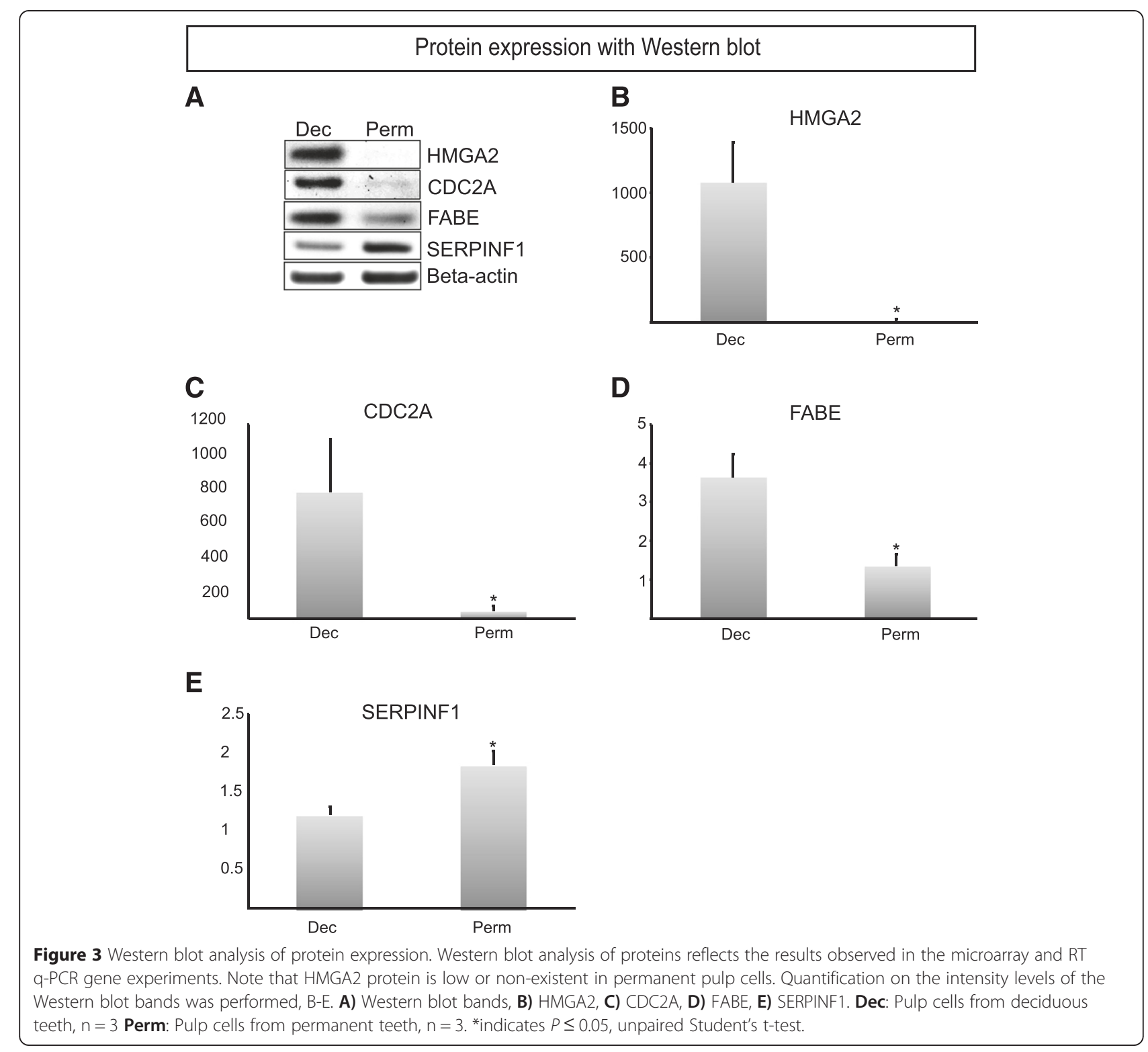


and division, including MAD2L1, MMP23A-MMP23B, MMP6 and PTTG, were highly expressed in pulp cells from deciduous teeth. Remarkably, $H M G A 2$, a gene strongly expressed by neural stem cells during embryogenesis, was highly expressed in pulp cells of deciduous but not permanent teeth (Figure $1 \mathrm{~B}$ and Table 3). The differences in mRNA expression are significant with the moderated T-test, $P \leq 0.05$.

IPA revealed that several genes are in association with $H M G A 2$, in particular genes that are involved with stemness, tumorigenesis and cell regulation, such as $S P 1$, $B R C A 1$ and cyclin A (Additional files 2 and 3).

To validate the microarray analysis, RT q-PCR was performed for genes of interest, especially those involved in cell proliferation and mitosis (Tables 2 and 3). This confirmed the microarray data obtained (Figure 2) with significant differences $(P \leq 0.05)$. For example, genes that are involved in cell cycle division and mitosis, such as $T K 1$, $C D K 4$ and $M M P 6$, were highly expressed in cells from deciduous teeth. We further probed the expression of proteins coded by selected genes that were detected by microarray and RT q-PCR. Western blot analysis and quantification demonstrated that HMGA2, FABE and CDC2A protein levels were elevated in cells from deciduous teeth while SERPINF1 had a higher expression in pulp cells from permanent teeth (Figure 3A-E).

siRNA-mediated loss-of-function experiments show association between HMGA2 and NANOG in pulp cells

To elucidate the importance of elevated levels of HMGA2 in deciduous teeth, we suppressed the HMGA2 levels by siRNA transfections in deciduous pulp cells and compared the expression of a stem cell marker in siControl (untreated samples) versus siHMGA2 treatments (Figure 4A). The results demonstrated that at 48 hours after transfection the expression of the stem cell gene NANOG was reduced (Figure 4B), although SOX2 and OCT4 expressions were unchanged. The graph shows standard error of the mean, normalized to the housekeeping gene GAPDH. The difference in mRNA expression between siControl versus siHMGA2 is significant with the Student's t-test, $P \leq 0.05$.

For comparisons of pluripotency markers, RT q-PCR levels of HMGA2, NANOG and OCT4 were determined simultaneously in decidous pulp cells, BMMSCs and human embryonic stem cells (HES). As expected, HES displayed very high levels of HMGA2, NANOG and OCT4 expression compared to deciduous pulp cells and BMMSCs. However, deciduous pulp cells and BMMSCs had similar expression levels with regard to these markers (Figure 5).

Immunohistochemistry reveals that HMGA2 expression in deciduous pulp tissue is unrelated to blood vessels

Sections from deciduous tooth pulps showed a marked nuclear HMGA2 immunoreactivity in stromal cells, while no corresponding immunostaining was observed in permanent pulp tissue (Figure 6A-G and Figure 7A-G). HMGA2 immunoreactivity was not omnipresent in deciduous pulps, but rather seemed to be selectively expressed in a subpopulation of cells (Figure 6C, E and G). The proportion of HMGA2-positive cells was quantified and found to be $16.86 \pm 2.28 \%$ (Additional file 4). The organization of deciduous and permanent tooth pulps, respectively, is displayed in Additional file 5. Further immunohistochemistry stainings with CD31 showed that HMGA2 expression does not co-localize with blood vessels (Figure 8).

\section{Immunohistochemistry reveals HMGA2 expression in embryonic gingiva}

HMGA2 nuclear immunoreactivity was abundant in cells of embryonic gingiva, in epithelial as well as in the
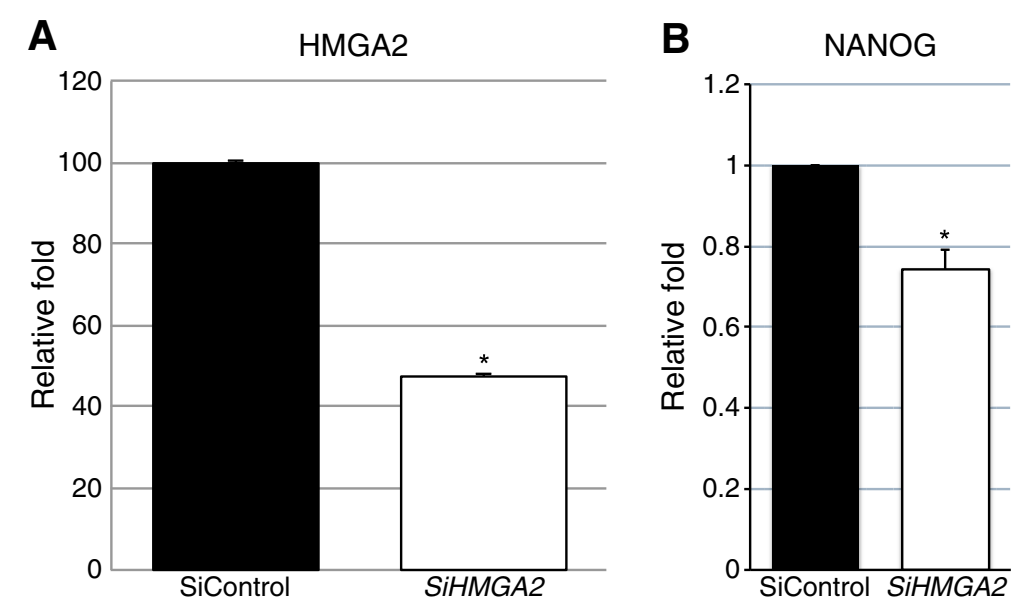

Figure 4 HMGA2 siRNA transfection of deciduous pulp cells in vitro. A) There is an endogenous reduction of HMGA2 mRNA levels compared to control conditions, $P \leq 0.05$. B) Endogenous reduction of HMGA2 mRNA levels reduces the expression of NANOG as compared to control conditions. *indicates $P \leq 0.05$, Student's t-test. 


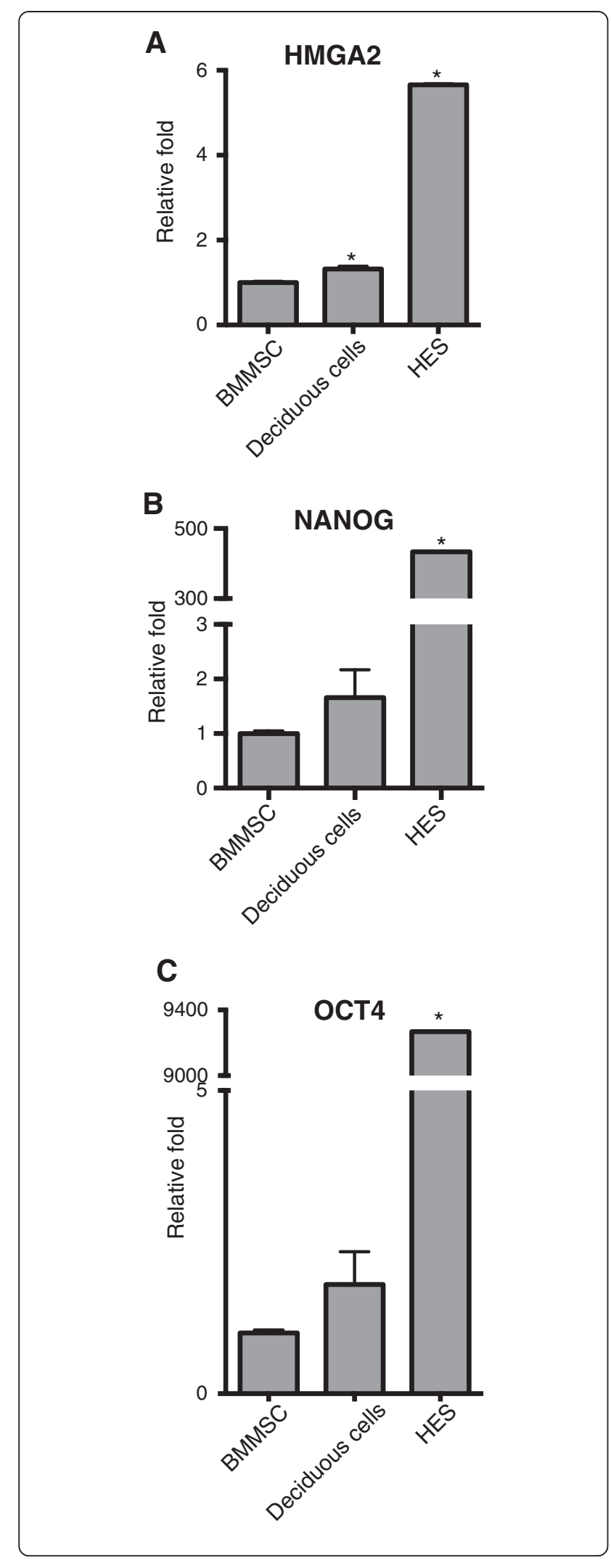

Figure 5 RT q-PCR of BMMSCs, deciduous pulp cells and HES. BMMSCs was set as the standard that deciduous pulp cells and HES were compared to with relative fold change. A) HMGA2 gene expression, B) NANOG gene expression, C) OCT4 gene expression. *indicates $P \leq 0.05$, Student's t-test. BMMSCs: bone marrow mesenchymal stem cells; HES: human embryonic stem cells.

mesenchymal compartments of $24.76 \pm 9.57 \%$ (Additional file 6). Adult gingival tissue did not express discernible HMGA2 protein immunostaining (Additional file 7).

\section{Discussion}

Humans are diphyodont and have two generations of teeth, where the second permanent set slowly begins to replace the first deciduous set around the age of six. Studies of molecular differences in pulpal cells from teeth extracted from children and adults, respectively, provide unique opportunities to compare general agerelated differences in human stromal populations. The regenerative capacity of human tissue is much greater during early childhood compared to later stages in life [24]. We hypothesized that this would be reflected in age-related changes in molecular characteristics of human pulpal stromal cells.

For this purpose, we compared gene expression profiles of pulp cells from deciduous and permanent teeth using a microarray chip that is specifically designed for stem/progenitor cells. This was performed to evaluate their genetic signatures with regard to key differences related to self-renewal, proliferation and differentiation. Cells from deciduous teeth were obtained from children (3- to 12-years old), thus being younger than the permanent teeth cells we collected from adults (18- to 52years old). For obvious reasons, there was no possibility to match deciduous and permanent teeth to the same donor, which is reflected in the microarray results (Figure 1A). However, with this taken into account, our results clearly show that there are differentially expressed genes in the deciduous and permanent teeth. Genes involved in cell division, mitosis, stemness and aging were differently expressed in favor of pulp cells from deciduous teeth. The results indicate a regeneration-favorable phenotype of pulp cells from deciduous teeth.

Somatic stem cells age with time, with a concomitant reduction in amounts, mitotic activity and self-renewable capacity $[1,25,26]$. This should be ascribed to age-dependent alterations in gene function. When mesenchymal cells from bone marrow were compared between adults and children, it was proposed that the bone marrow cells from the latter are more suitable for tissue engineering purposes because of age-related factors [3]. HMGA2 is an example of a gene that confers self-renewal and proliferation capacity to stem cells. It is strongly expressed by neural stem cells/progenitors in 


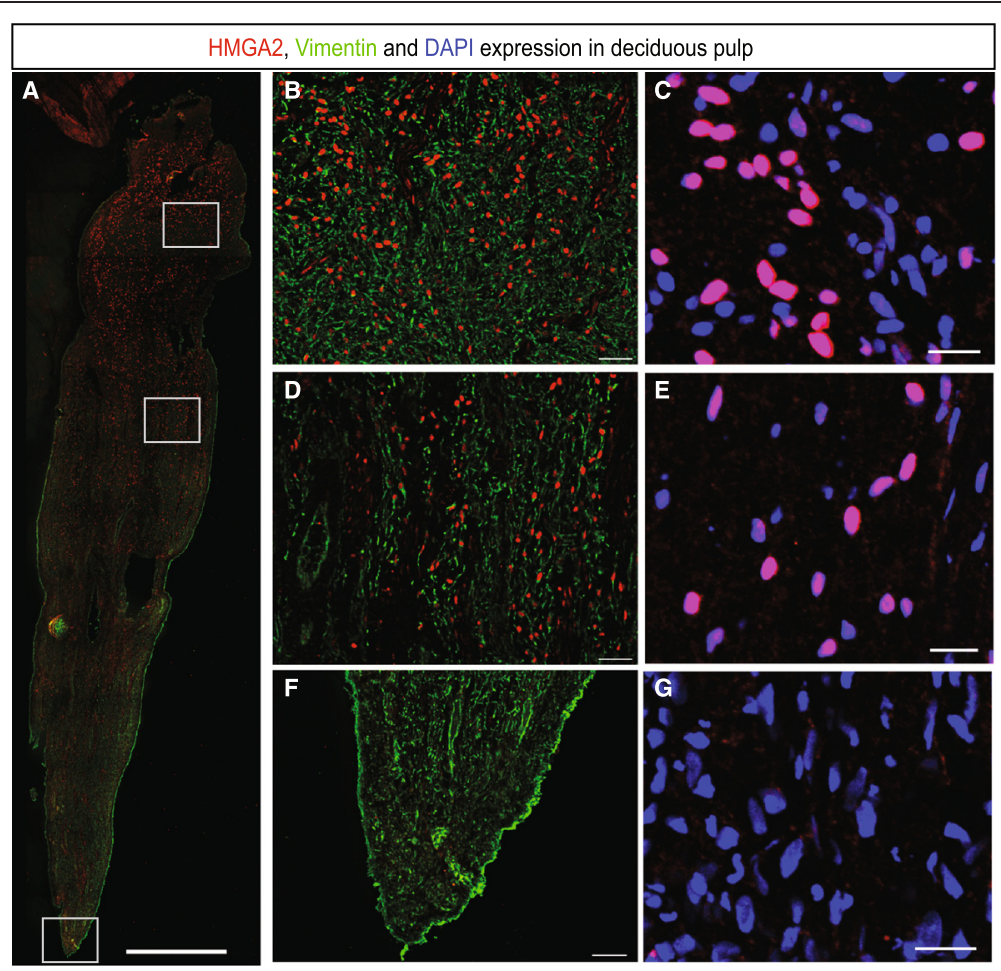

Figure 6 HMGA2 expression in deciduous pulp. HMGA2 is abundantly expressed in deciduous pulp but only by a subpopulation of cells. A) HMGA2 (red), vimentin (green). B-G are zoomed in areas. C, E, G show co-localization of DAPI staining and HMGA2. A: scale bar 1,000 $\mu$ m; B, D and F: $50 \mu \mathrm{m} ; \mathrm{C}, \mathrm{E}$ and G: scale bar $20 \mu \mathrm{m}$. DAPI, 4',6-diamidino-2-phenylindole.

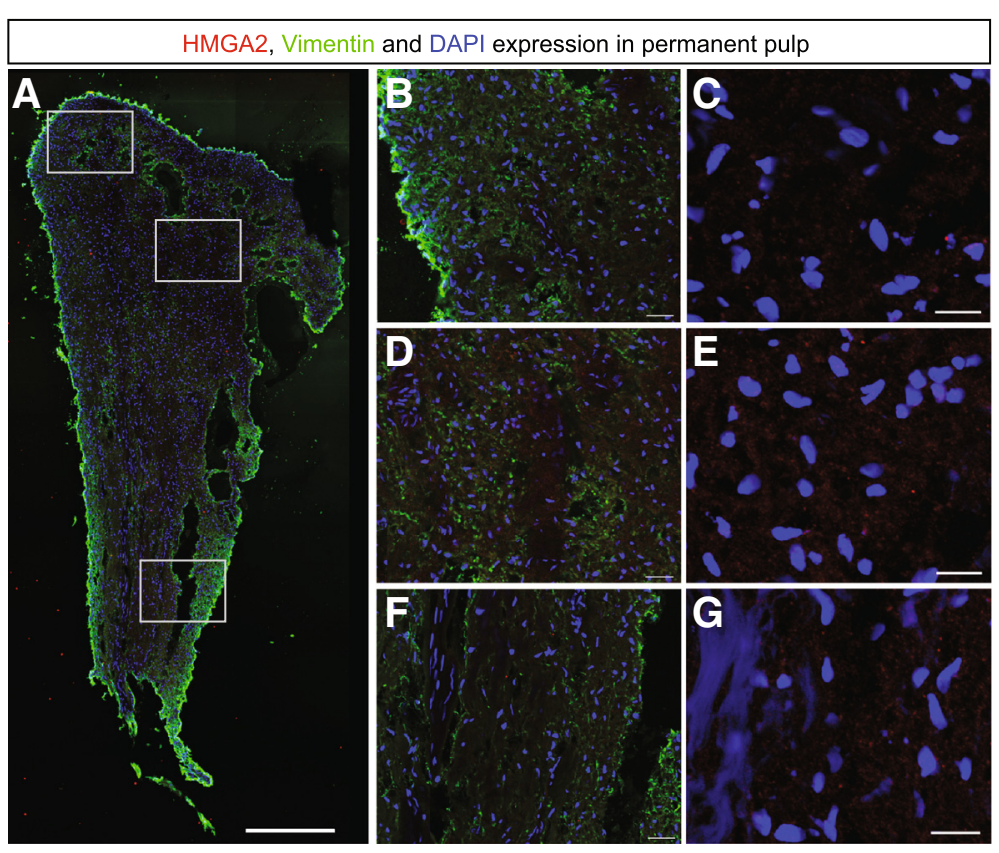

Figure 7 HMGA2 expression in permanent pulp. HMGA2 expression is low or non-existent in permanent pulp. A) HMGA2 (red), vimentin (green) and

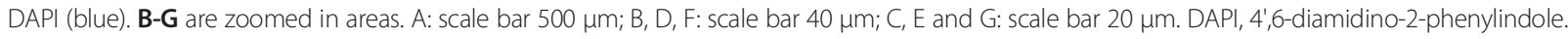




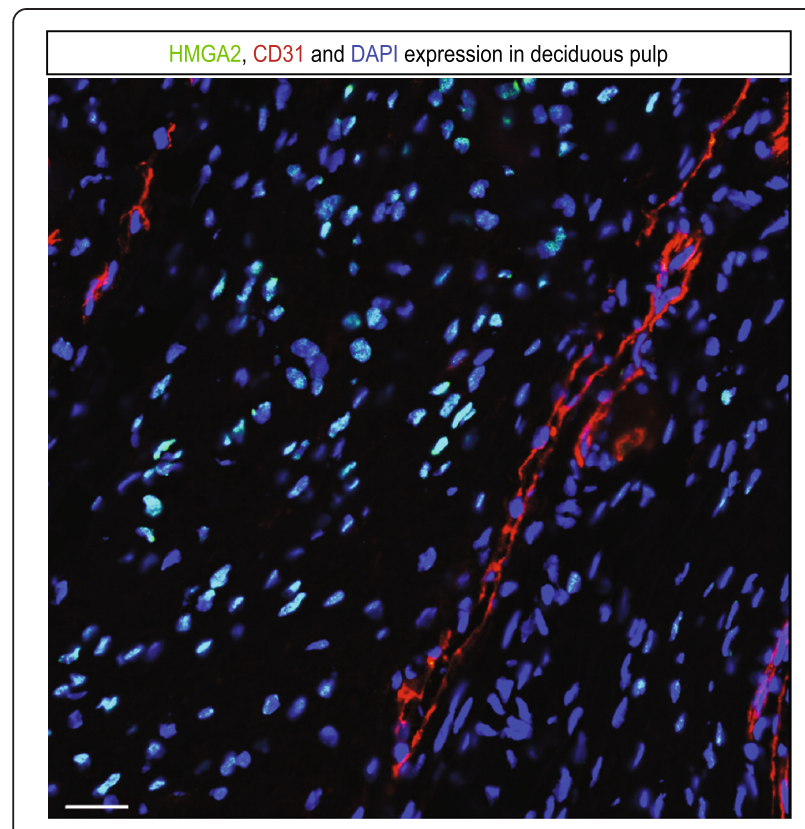

Figure $\mathbf{8}$ Immunohistochemistry of HMGA2 expression and blood vessels in deciduous pulp. HMGA2 does not co-localize with blood vessels in deciduous tooth pulp, HMGA2 (green), CD31 (red) and DAPI (blue). Scale bar $30 \mu \mathrm{m}$. DAPI, 4',6-diamidino-2-phenylindole.

the subventricular zone, but declines over time with age [27-29]. A recent study has shown that the senescencerelated tumor suppressor gene $p 16^{\text {Ink4a }}$, which diminishes the self renewal capacity of cells and also reduces the expression of $H M G A 2$, is increased in older permanent teeth compared to young deciduous teeth [30]. This agrees with our data, where HMGA2 was differentially expressed with a substantial level in cells from deciduous teeth but not detected in cells from permanent pulps. An overall agedependent decline in cellular expression of HMGA2 outside brain and teeth is suggested by our immunohistochemical observations in human gingiva, where embryonic tissue expressed widespread HMGA2 immunoreactivity while none was observed in adult samples.

It should be noted, that our results show that not all stromal cells in deciduous pulps are HMGA2 positive. Instead, $\mathrm{HMGA}^{+}$cells constitute a subpopulation of approximately $16 \%$ of the total number. The full phenotype/s of this subpopulation remains elusive, although the HMGA2 expression suggests that it may contain progenitor cells. Other investigators have demonstrated that stem/progenitor cells in teeth are associated with blood vessels $[7,12]$. We did not find that HMGA2-positive cells were related to blood vessels, which suggests that we might have found another proliferative subpopulation in deciduous teeth.

We attempted to clarify the role of HMGA2 in deciduous teeth pulp cells by reducing the level through siRNA transfections. Due to stable expression of HMGA2 mRNA in the cells, we managed to suppress HMGA2 levels to approximately $50 \%$ of those siControl untreated samples (Figure 4A). This resulted in a significant reduction of mRNA levels of NANOG, which is thought to be a key factor in maintaining pluripotency. This finding gives further indications that HMGA2 has a key role in the regulation and maintenance of a subset of pulpal cells. SOX2 levels were not detectable, which is expected since dental stem cells that express $S O X 2$ are epithelial and not mesenchymal. OCT4 levels were not affected by the depletion of HMGA2.

Our results indicate that deciduous teeth harbor a population of cells, with a higher self-renewal and proliferation capacity than those of permanent teeth. Thus, markers involved in cell division and mitosis were up-regulated in deciduous cells compared to cells from permanent teeth (Table 2). This could make primary pulp cells more suitable for tissue regeneration. In fact, from certain aspects, they might even be more useful than BMMSCs, since the levels of stem cell markers NANOG and OCT4 were similar between these cell types, but HMGA2 was slightly higher in deciduous pulp cells (Figure 5A-C).

\section{Conclusions}

Using specifically designed microarray panels, RT qPCR, Western blot and immunohistochemistry we show that HMGA2, a stem cell-associated gene, is robustly expressed in deciduous pulp cells but not in permanent tooth pulp cells. Additionally, several genes associated with mitosis were highly expressed in deciduous pulp cells, while matrix genes and several signaling molecules were strongly expressed in the adult. These findings strongly suggest that there are molecular differences in cellular populations of different age and should serve as important basic information for understanding the therapeutic potential of pulpal cells. Specifically, primary pulp cells may be better suited for regenerative medicine and tissue engineering purposes than cells from permanent tooth pulps and based on this, are recommended for early banking.

\section{Additional files}

Additional file 1: The 70 differently expressed genes in microarray, $P \leq 0.05$.

Additional file 2: Network representation of HMGA2 interacting genes in permanent compared to deciduous tooth. Direct interacting, highly interconnected genes merged from Ingenuity Pathway analysis.

Genes are represented as nodes and relations between genes, interactions, as lines. Gene modulation direction as a result of the comparison is color coded: green, down-regulated and red, up-regulated and color intensity represents strength of the modulation. Node shapes represent different gene classes.

Additional file 3: Network representation of interacting genes in adult compared to deciduous tooth. Top ranking networks of directly interacting, highly interconnected genes merged from Ingenuity Pathway analysis. Genes are represented as nodes and relations between genes, 
interactions, as lines. Gene modulation direction as a result of the comparison is color coded: green, down-regulated and red, up-regulated and color intensity represents strength of the modulation. Node shapes represent different gene classes. Genes are placed according to their subcellular localization.

Additional file 4: Immunofluorescence intensity of HMGA2. The immunofluorescence intensity was measured in HMGA2+ cells and negative control cells.

Additional file 5: Hematoxylin \& Eosin staining of deciduous and permanent tooth pulps. The H\&E staining shows the organization of tooth pulp. A) Deciduous tooth pulp, B) Permanent tooth pulp. Scale bar $200 \mu \mathrm{m}$.

Additional file 6: Immunohistochemistry of HMGA2 expression in embryonic gingiva. HMGA2 is abundantly expressed in epithelial and mesenchymal cells of embryonic gingiva, and is co-localized with nuclear DAPI staining. A) HMGA2 (green), CD31 (a blood vessel marker) (red) and DAPI (blue). B-E are zoomed in areas. Scale bars. A $70 \mu \mathrm{m} ; \mathrm{B}$-E: $30 \mu \mathrm{m}$.

Additional file 7: Immunohistochemistry of HMGA2 expression in adult gingiva. HMGA2 expression is not observed in adult gingiva. A) HMGA2 (green), CD31 (a blood vessel marker) (red) and DAPI (blue). B-E are zoomed in areas. Scale bars: A $500 \mu \mathrm{m}$; B-C: $50 \mu \mathrm{m}$; D-E: $70 \mu \mathrm{m}$.

\section{Abbreviations}

BMMSCs: bone marrow mesenchymal stem cells; HES: human embryonic stem cells; HMGA2: high-mobility group AT-hook 2; Ig: immunoglobulin; IPA: Ingenuity Pathway Analysis; OCT: optimum cutting temperature; PBS: phosphate-buffered saline; RT q-PCR: real time quantitative real-time polymerase chain reaction; siRNA: small interfering RNA.

\section{Competing interests}

The authors declare that they have no competing interests.

\section{Authors' contributions}

NK, MC, JJM, IA and KF participated in conceiving and designing the study. NK collected tissue, performed cell culture work, RT q-PCR, Western blot and immunohistochemistry experiments. MD performed siRNA mediated knock down experiments. TYL collected adult gingiva biopsies and sectioned them. ES collected embryonic gingiva. MLL performed cell culture and RT q-PCR of HES and BMMSC. NK, MC, MLL, IA and PG analyzed data. NK, PG, MD, TYL, $M L L, I A, J J M$ and KF wrote the paper. All authors read and approved the final manuscript.

\section{Acknowledgements}

This study has been supported by the Swedish Dental Society and we would also like to thank Knut and Alice Wallenberg Foundation (CLICK facility). Work in this manuscript is based on a PhD dissertation by Nina Kaukua at the Karolinska Institutet, with parts of experiments performed at Columbia University. We thank Ms. R. Burdie, Q. Guo, H. Keyes and Ms. J. Melendez, R. Bergström-Tengzelius, F. Holm, M. Barr Agholme, A.-M. Thelander, M-L Olsson and S. Kaukua for their technical and administrative assistance.

\section{Author details}

${ }^{1}$ Center for Craniofacial Regeneration (CCR), Columbia University Medical Center, 630 W. 168 St. - PH7E, New York, NY 10032, USA. ²Department of Neuroscience, Karolinska Institutet, Stockholm SE-171 77, Sweden. ${ }^{3}$ Department of Systems Biology, Herbert Irving Comprehensive Cancer Center, Columbia University Medical Center, New York, NY 10032, USA. ${ }^{4}$ Department of Dental Medicine, Karolinska Institutet, Huddinge SE 14104, Sweden. ${ }^{5}$ Advanced Center for Translational Regenerative Medicine (ACTREM), Department for Clinical Science, Intervention and Technology (CLINTEC), Division of Ear, Nose and Throat, Karolinska Institutet, Huddinge SE 141 86, Sweden. 'Division of Neurodegeneration, Department of Neurobiology, Care Sciences and Society, Karolinska Institutet, Huddinge SE-14157, Sweden. 7Department of Physiology and Pharmacology, Karolinska Institutet, Stockholm SE 171 77, Sweden.

Received: 15 May 2014 Revised: 9 June 2014

Accepted: 18 March 2015 Published online: 18 April 2015

\section{References}

1. Enwere E, Shingo T, Gregg C, Fujikawa H, Ohta S, Weiss S. Aging results in reduced epidermal growth factor receptor signaling, diminished olfactory neurogenesis, and deficits in fine olfactory discrimination. J Neurosci. 2004;24:8354-65.

2. Sharpless NE, DePinho RA. How stem cells age and why this makes us grow old. Nat Rev Mol Cell Biol. 2007;8:703-13.

3. Choumerianou DM, Martimianaki G, Stiakaki E, Kalmanti L, Kalmanti M, Dimitriou $\mathrm{H}$. Comparative study of stemness characteristics of mesenchymal cells from bone marrow of children and adults. Cytotherapy. 2010;12:881-7.

4. Jung Y, Brack AS. Cellular mechanisms of somatic stem cell aging. Curr Top Dev Biol. 2014;107:405-38.

5. Gronthos S, Mankani M, Brahim J, Robey PG, Shi S. Postnatal human dental pulp stem cells (DPSCs) in vitro and in vivo. Proc Natl Acad Sci U S A. 2000;97:13625-30.

6. Shi S, Robey PG, Gronthos S. Comparison of human dental pulp and bone marrow stromal stem cells by cDNA microarray analysis. Bone. 2001;29:532-9.

7. Miura M, Gronthos S, Zhao M, Lu B, Fisher LW, Robey PG, et al. SHED: stem cells from human exfoliated deciduous teeth. Proc Natl Acad Sci U S A. 2003;100:5807-12.

8. Sakai K, Yamamoto A, Matsubara K, Nakamura S, Naruse M, Yamagata M, et al. Human dental pulp derived stem cells promote locomotor recovery after complete transection of the rat spinalcord by multiple neuro regenerative mechanisms. J Clin Invest. 2012;3:80-90.

9. Yang R, Chen M, Lee CH, Yoon R, Lal S, Mao JJ. Clones of ectopic stem cells in the regeneration of muscle defects in vivo. PLoS One. 2010;5, e13547.

10. Ma L, Makino Y, Yamaza H, Akiyama K, Hoshino Y, Nonaka K, et al. Cryopreserved dental pulp tissues of exfoliated deciduous teeth is a feasible stem cell resource for regenerative medicine. PLoS One. 2012;7:12.

11. Smith AJ, Smith JG, Shelton RM, Cooper PR. Harnessing the natural regenerative potential of the dental pulp. Dent Clin North Am. 2012;56:589-601.

12. Huang GT, Gronthos S, Shi S. Mesenchymal stem cells derived from dental tissues vs. those from other sources: their biology and role in regenerative medicine. J Dent Res. 2009;88:792-806.

13. Menicanin D, Bartold PM, Zannettino AC, Gronthos S. Identification of a common gene expression signature associated with immature clonal mesenchymal cell populations derived from bone marrow and dental tissues. Stem Cells Dev. 2010;19:1501-10.

14. Gronthos S, Brahim J, Li W, Fisher LW, Cherman N, Boyde A, et al. Stem cell properties of human dental pulp stem cells. J Dent Res. 2002;81:531-5.

15. Tete S, Mastrangelo F, Scioletti AP, Tranasi M, Raicu F, Paolantonio M, et al. Microarray expression profiling of human dental pulp from single subject. Clin Invest Med. 2008;31:e55-61.

16. Tranasi M, Sberna MT, Zizzari V, D'Apolito G, Mastrangelo F, Salini L, et al. Microarray evaluation of age-related changes in human dental pulp. J Endod. 2009;35:1211-7.

17. Govindasamy V, Abdullah AN, Ronald VS, Musa S, Ab Aziz ZA, Nain RB, et al. Inherent differential propensity of dental pulp stem cells derived from human deciduous and permanent teeth. J Endod. 2010;36:1504-15.

18. Suzuki T, Lee CH, Chen M, Zhao W, Fu SY, Qi JJ, et al. Induced migration of dental pulp stem cells for in vivo pulp regeneration. J Dent Res. 2011;90:1013-8.

19. Smyth G. Limma: linear models for microarray data. In: Gentleman R, Carey V, Huber W, Irizarry R, Dudoit S, editors. Bioinformatics and computational biology solutions using R and Bioconductor. Berlin: Springer-Verlag; 2005. p. 397-420.

20. Huang DW, Sherman BT, Lempicki RA. Systematic and integrative analysis of large gene lists using DAVID Bioinformatics Resources. Nature Protoc. 2009:4:44-57.

21. Huang DW, Sherman BT, Lempicki RA. Bioinformatics enrichment tools: paths toward the comprehensive functional analysis of large gene lists. Nucleic Acids Res. 2009;37:1-13.

22. Merico D, Isserlin R, Bader GD. Visualizing gene-set enrichment results using the Cytoscape plug-in enrichment map. Methods Mol Biol. 2011;781:257-77.

23. Shannon P, Markiel A, Ozier O, Baliga NS, Wang JT, Ramage D, et al. Cytoscape: a software environment for integrated models of biomolecular interaction networks. Genome Res. 2003;13:2498-504.

24. Illingworth CM. Trapped fingers and amputated finger tips in children. J Pediatr Surg. 1974;9:853-8. 
25. Maslov AY, Barone TA, Plunkett RJ, Pruitt SC. Neural stem cell detection, characterization, and age-related changes in the subventricular zone of mice. J Neurosci. 2004;24:1726-33.

26. Molofsky AV, Pardal R, Iwashita T, Park IK, Clarke MF, Morrison SJ. Bmi-1 dependence distinguishes neural stem cell self-renewal from progenitor proliferation. Nature. 2003:425:962-7.

27. Nishino J, Kim I, Chada K, Morrison SJ. Hmga2 promotes neural stem cell self-renewal in young but not old mice by reducing p16lnk4a and p19Arf expression. Cell. 2008;135:227-39.

28. Hammond SM, Sharpless NE. HMGA2, microRNAs, and stem cell aging. Cell. 2008;135:1013-6.

29. Ashar HR, Chouinard Jr RA, Dokur M, Chada K. In vivo modulation of HMGA2 expression. Biochim Biophys Acta. 2010;1799:55-61.

30. Feng X, Xing J, Feng G, Huang D, Lu X, Liu S, et al. p16INK4A mediates age-related changes in mesenchymal stem cells derived from human dental pulp through the DNA damage and stress response. Mech Ageing Dev. 2014;141-142:46-55. doi:10.1016/j.mad.2014.09.004

\section{Submit your next manuscript to BioMed Central and take full advantage of:}

- Convenient online submission

- Thorough peer review

- No space constraints or color figure charges

- Immediate publication on acceptance

- Inclusion in PubMed, CAS, Scopus and Google Scholar

- Research which is freely available for redistribution 